\title{
Storage of Methane in Clathrate Hydrates: Monte Carlo Simulations of sI Hydrates and Comparison with Experimental Measurements
}

\section{Supporting Information}

\author{
Nikolaos I. Papadimitriou ${ }^{1}$, Ioannis N. Tsimpanogiannis ${ }^{1,2, *}$, \\ Ioannis G. Economou ${ }^{2, *}$ and Athanassios K. Stubos ${ }^{1}$ \\ ${ }^{1}$ National Center for Scientific Research "Demokritos", \\ Environmental Research Laboratory, \\ 15310 Aghia Paraskevi Attikis, Greece \\ ${ }^{2}$ Texas A\&M University at Qatar, Chemical Engineering Program, \\ Education City, PO Box 23874, Doha, Qatar
}

*Corresponding authors at: i.tsimpanogiannis@qatar.tamu.edu (Ioannis N. Tsimpanogiannis) and ioannis.economou@qatar.tamu.edu (Ioannis G. Economou) 


\section{Monte Carlo Results}

Each uncertainty was estimated at the confidence limit (95\%).

\begin{tabular}{|ccc|cc|cc|}
\hline \multicolumn{6}{|c|}{ Table S1. OPLS - UA } & \multicolumn{2}{c|}{ Uncertainty, $\boldsymbol{u}$} \\
\cline { 1 - 3 } $\boldsymbol{T}(\mathbf{K})$ & $\boldsymbol{P}(\mathbf{M P a})$ & $\boldsymbol{\mu} / \boldsymbol{k}_{b}(\mathbf{K})$ & $\boldsymbol{\theta}_{\text {Small }}$ & $\boldsymbol{\theta}_{\text {Large }}$ & \multirow{2}{*}{$\boldsymbol{\theta}_{\text {Small }}$} & $\boldsymbol{\theta}_{\text {Large }}$ \\
\hline 200 & 0.100 & -2752.4 & 0.9775 & 0.9870 & 0.0009 & 0.0007 \\
200 & 0.200 & -2614.7 & 0.9881 & 0.9934 & 0.0007 & 0.0005 \\
200 & 0.502 & -2434.5 & 0.9958 & 0.9973 & 0.0004 & 0.0003 \\
200 & 1.010 & -2301.0 & 0.9978 & 0.9986 & 0.0003 & 0.0002 \\
200 & 2.038 & -2173.1 & 0.9990 & 0.9993 & 0.0002 & 0.0002 \\
200 & 5.391 & -2027.4 & 0.9993 & 0.9997 & 0.0002 & 0.0001 \\
200 & 12.374 & -1971.1 & 0.9995 & 0.9998 & 0.0001 & 0.0001 \\
200 & 23.711 & -1896.4 & 0.9998 & 0.9998 & 0.0001 & 0.0001 \\
200 & 56.300 & -1716.1 & 0.9999 & 0.9999 & 0.0001 & 0.0000 \\
200 & 82.010 & -1594.8 & 1.0000 & 1.0000 & 0.0000 & 0.0000 \\
200 & 106.861 & -1456.2 & 1.0000 & 1.0000 & 0.0000 & 0.0000 \\
200 & 137.455 & -1339.1 & 1.0000 & 1.0000 & 0.0000 & 0.0000 \\
200 & 179.156 & -1156.7 & 1.0000 & 1.0000 & 0.0000 & 0.0000 \\
200 & 207.510 & -1032.3 & 1.0000 & 1.0000 & 0.0000 & 0.0000 \\
\hline
\end{tabular}

\begin{tabular}{|ccc|cc|cc|}
\hline \multicolumn{6}{|c|}{ Table S2. OPLS $-\mathbf{A A}$} & \multicolumn{2}{c|}{ Uncertainty, $\boldsymbol{u}$} \\
\cline { 1 - 3 } $\boldsymbol{T}(\mathbf{K})$ & $\boldsymbol{P}(\mathbf{M P a})$ & $\boldsymbol{\mu} / \boldsymbol{k}_{b}(\mathbf{K})$ & $\boldsymbol{\theta}_{\text {Small }}$ & $\boldsymbol{\theta}_{\text {Large }}$ & \multirow{2}{*}{$\boldsymbol{\theta}_{\text {Small }}$} & $\boldsymbol{\theta}_{\text {Large }}$ \\
\hline 200 & 0.100 & -2613.6 & 0.9688 & 0.9778 & 0.0011 & 0.0009 \\
200 & 0.201 & -2475.8 & 0.9854 & 0.9890 & 0.0007 & 0.0006 \\
200 & 0.503 & -2295.2 & 0.9942 & 0.9955 & 0.0005 & 0.0004 \\
200 & 1.011 & -2161.1 & 0.9970 & 0.9977 & 0.0003 & 0.0003 \\
200 & 2.052 & -2031.5 & 0.9983 & 0.9988 & 0.0003 & 0.0002 \\
200 & 5.539 & -1879.1 & 0.9994 & 0.9995 & 0.0002 & 0.0001 \\
200 & 10.623 & -1830.9 & 0.9994 & 0.9995 & 0.0002 & 0.0001 \\
200 & 17.947 & -1784.5 & 0.9995 & 0.9997 & 0.0001 & 0.0001 \\
200 & 41.447 & -1659.5 & 0.9997 & 0.9998 & 0.0001 & 0.0001 \\
200 & 60.797 & -1564.0 & 0.9998 & 0.9999 & 0.0001 & 0.0001 \\
200 & 79.969 & -1454.5 & 0.9999 & 0.9999 & 0.0000 & 0.0001 \\
200 & 102.601 & -1333.1 & 1.0000 & 1.0000 & 0.0000 & 0.0000 \\
200 & 137.901 & -1182.1 & 1.0000 & 1.0000 & 0.0000 & 0.0000 \\
200 & 161.065 & -1085.5 & 1.0000 & 1.0000 & 0.0000 & 0.0000 \\
\hline
\end{tabular}




\begin{tabular}{|c|c|c|c|c|c|c|}
\hline \multicolumn{5}{|c|}{ Table S3. OPLS - UA } & \multicolumn{2}{|c|}{ Uncertainty, $u$} \\
\hline$T(\mathbf{K})$ & $P$ (MPa) & $\boldsymbol{\mu} / \boldsymbol{k}_{b}(\mathbf{K})$ & $\theta_{\text {Small }}$ & $\theta_{\text {Large }}$ & $\theta_{\text {Small }}$ & $\theta_{\text {Large }}$ \\
\hline 250 & 0.100 & -3579.3 & 0.6275 & 0.7624 & 0.0030 & 0.0026 \\
\hline 250 & 0.200 & -3406.7 & 0.7954 & 0.8808 & 0.0025 & 0.0020 \\
\hline 250 & 0.501 & -3179.5 & 0.9104 & 0.9531 & 0.0018 & 0.0013 \\
\hline 250 & 1.005 & -3009.4 & 0.9540 & 0.9762 & 0.0013 & 0.0009 \\
\hline 250 & 2.018 & -2842.6 & 0.9767 & 0.9874 & 0.0009 & 0.0007 \\
\hline 250 & 5.127 & -2633.6 & 0.9889 & 0.9947 & 0.0007 & 0.0005 \\
\hline 250 & 10.537 & -2495.6 & 0.9942 & 0.9970 & 0.0005 & 0.0003 \\
\hline 250 & 21.603 & -2378.0 & 0.9965 & 0.9980 & 0.0004 & 0.0003 \\
\hline 250 & 54.524 & -2160.5 & 0.9986 & 0.9991 & 0.0002 & 0.0002 \\
\hline 250 & 80.338 & -2010.4 & 0.9991 & 0.9997 & 0.0002 & 0.0001 \\
\hline 250 & 105.873 & -1871.7 & 0.9993 & 0.9998 & 0.0002 & 0.0001 \\
\hline 250 & 135.933 & -1715.6 & 0.9996 & 0.9999 & 0.0001 & 0.0001 \\
\hline 250 & 177.366 & -1520.9 & 0.9998 & 0.9999 & 0.0001 & 0.0001 \\
\hline 250 & 205.991 & -1404.3 & 1.0000 & 1.0000 & 0.0000 & 0.0000 \\
\hline
\end{tabular}

\begin{tabular}{|ccc|cc|cc|}
\hline \multicolumn{6}{|c|}{ Table S4. OPLS - AA } & \multicolumn{2}{c|}{ Uncertainty, $\boldsymbol{u}$} \\
\cline { 1 - 3 } $\boldsymbol{T}(\mathbf{K})$ & $\boldsymbol{P}(\mathbf{M P a})$ & $\boldsymbol{\mu} / \boldsymbol{k}_{b}(\mathbf{K})$ & $\boldsymbol{\theta}_{\text {Small }}$ & $\boldsymbol{\theta}_{\text {Large }}$ & \multirow{\boldsymbol{\theta}_{\text{Small}}}{*}{} & $\boldsymbol{\theta}_{\text {Large }}$ \\
\hline 250 & 0.100 & -3405.9 & 0.5456 & 0.6719 & 0.0031 & 0.0029 \\
250 & 0.200 & -3233.1 & 0.7342 & 0.8223 & 0.0027 & 0.0024 \\
250 & 0.502 & -3005.4 & 0.8784 & 0.9251 & 0.0020 & 0.0016 \\
250 & 1.007 & -2834.5 & 0.9373 & 0.9625 & 0.0015 & 0.0012 \\
250 & 2.031 & -2666.0 & 0.9669 & 0.9807 & 0.0011 & 0.0009 \\
250 & 5.211 & -2451.8 & 0.9868 & 0.9917 & 0.0007 & 0.0006 \\
250 & 10.895 & -2304.7 & 0.9919 & 0.9954 & 0.0006 & 0.0004 \\
250 & 21.335 & -2185.2 & 0.9952 & 0.9971 & 0.0004 & 0.0003 \\
250 & 49.116 & -1995.7 & 0.9974 & 0.9988 & 0.0003 & 0.0002 \\
250 & 70.585 & -1871.4 & 0.9989 & 0.9992 & 0.0002 & 0.0002 \\
250 & 94.445 & -1760.0 & 0.9993 & 0.9995 & 0.0002 & 0.0001 \\
250 & 119.517 & -1628.7 & 0.9994 & 0.9997 & 0.0002 & 0.0001 \\
250 & 155.155 & -1449.0 & 0.9997 & 0.9999 & 0.0001 & 0.0001 \\
250 & 182.129 & -1328.8 & 0.9998 & 0.9999 & 0.0001 & 0.0001 \\
\hline
\end{tabular}




\begin{tabular}{|c|c|c|c|c|c|c|}
\hline \multicolumn{5}{|c|}{ Table S5. OPLS - UA } & \multicolumn{2}{|c|}{ Uncertainty, $u$} \\
\hline$T(\mathbf{K})$ & $P$ (MPa) & $\boldsymbol{\mu} / \boldsymbol{k}_{b}(\mathbf{K})$ & $\theta_{\text {Small }}$ & $\theta_{\text {Large }}$ & $\theta_{\text {Small }}$ & $\theta_{\text {Large }}$ \\
\hline 270 & 0.100 & -3917.5 & 0.3647 & 0.5252 & 0.0030 & 0.0031 \\
\hline 270 & 0.200 & -3730.8 & 0.5745 & 0.7265 & 0.0031 & 0.0028 \\
\hline 270 & 0.501 & -3485.0 & 0.8025 & 0.8868 & 0.0025 & 0.0020 \\
\hline 270 & 1.004 & -3300.5 & 0.8913 & 0.9428 & 0.0019 & 0.0014 \\
\hline 270 & 2.015 & -3118.7 & 0.9443 & 0.9709 & 0.0014 & 0.0010 \\
\hline 270 & 5.097 & -2887.7 & 0.9724 & 0.9874 & 0.0010 & 0.0007 \\
\hline 270 & 10.374 & -2727.9 & 0.9862 & 0.9930 & 0.0007 & 0.0005 \\
\hline 270 & 21.155 & -2584.9 & 0.9920 & 0.9960 & 0.0006 & 0.0004 \\
\hline 270 & 53.688 & -2345.3 & 0.9966 & 0.9983 & 0.0004 & 0.0003 \\
\hline 270 & 79.143 & -2186.2 & 0.9983 & 0.9990 & 0.0003 & 0.0002 \\
\hline 270 & 105.497 & -2043.4 & 0.9989 & 0.9995 & 0.0002 & 0.0001 \\
\hline 270 & 136.936 & -1884.4 & 0.9994 & 0.9997 & 0.0001 & 0.0001 \\
\hline 270 & 177.535 & -1697.1 & 0.9995 & 0.9998 & 0.0001 & 0.0001 \\
\hline 270 & 206.554 & -1546.1 & 0.9998 & 0.9999 & 0.0001 & 0.0001 \\
\hline
\end{tabular}

\begin{tabular}{|c|c|c|c|c|c|c|}
\hline \multicolumn{5}{|c|}{ Table S6. OPLS - AA } & \multicolumn{2}{|c|}{ Uncertainty, $u$} \\
\hline$T(\mathbf{K})$ & $P(\mathbf{M P a})$ & $\boldsymbol{\mu} / \boldsymbol{k}_{b}(\mathbf{K})$ & $\theta_{\text {Small }}$ & $\theta_{\text {Large }}$ & $\theta_{\text {Small }}$ & $\theta_{\text {Large }}$ \\
\hline 270 & 0.100 & -3730.2 & 0.2961 & 0.4332 & 0.0028 & 0.0031 \\
\hline 270 & 0.200 & -3543.4 & 0.4926 & 0.6357 & 0.0031 & 0.0030 \\
\hline 270 & 0.501 & -3297.1 & 0.7330 & 0.8331 & 0.0027 & 0.0023 \\
\hline 270 & 1.006 & -3111.8 & 0.8533 & 0.9141 & 0.0022 & 0.0017 \\
\hline 270 & 2.027 & -2928.2 & 0.9225 & 0.9559 & 0.0017 & 0.0013 \\
\hline 270 & 5.168 & -2692.1 & 0.9680 & 0.9814 & 0.0011 & 0.0008 \\
\hline 270 & 10.761 & -2523.7 & 0.9819 & 0.9903 & 0.0008 & 0.0006 \\
\hline 270 & 22.025 & -2372.6 & 0.9899 & 0.9943 & 0.0006 & 0.0005 \\
\hline 270 & 50.986 & -2155.0 & 0.9956 & 0.9975 & 0.0004 & 0.0003 \\
\hline 270 & 69.697 & -2045.3 & 0.9966 & 0.9983 & 0.0004 & 0.0003 \\
\hline 270 & 97.117 & -1899.0 & 0.9980 & 0.9991 & 0.0003 & 0.0002 \\
\hline 270 & 122.338 & -1752.5 & 0.9989 & 0.9994 & 0.0002 & 0.0001 \\
\hline 270 & 160.244 & -1580.0 & 0.9995 & 0.9997 & 0.0001 & 0.0001 \\
\hline 270 & 187.855 & -1448.8 & 0.9997 & 0.9998 & 0.0001 & 0.0001 \\
\hline
\end{tabular}




\begin{tabular}{|ccc|cc|cc|}
\hline \multicolumn{6}{|c|}{ Table S7. OPLS - UA } & \multicolumn{2}{c|}{ Uncertainty, $\boldsymbol{u}$} \\
\cline { 1 - 3 } $\boldsymbol{T}(\mathbf{K})$ & $\boldsymbol{P}(\mathbf{M P a})$ & $\boldsymbol{\mu} / \boldsymbol{k}_{b}(\mathbf{K})$ & $\boldsymbol{\theta}_{\text {Small }}$ & $\boldsymbol{\theta}_{\text {Large }}$ & $\boldsymbol{\theta}_{\text {Small }}$ & $\boldsymbol{\theta}_{\text {Large }}$ \\
\hline 300 & 0.100 & -4431.6 & 0.1304 & 0.2388 & 0.0021 & 0.0026 \\
300 & 0.200 & -4224.1 & 0.2586 & 0.4163 & 0.0027 & 0.0031 \\
300 & 0.501 & -3950.3 & 0.5192 & 0.6894 & 0.0031 & 0.0029 \\
300 & 1.003 & -3744.4 & 0.7085 & 0.8331 & 0.0028 & 0.0023 \\
300 & 2.010 & -3540.4 & 0.8343 & 0.9141 & 0.0023 & 0.0017 \\
300 & 5.060 & -3277.6 & 0.9285 & 0.9642 & 0.0016 & 0.0012 \\
300 & 10.241 & -3089.0 & 0.9604 & 0.9810 & 0.0012 & 0.0008 \\
300 & 20.814 & -2911.8 & 0.9790 & 0.9897 & 0.0009 & 0.0006 \\
300 & 52.474 & -2638.0 & 0.9895 & 0.9957 & 0.0006 & 0.0004 \\
300 & 79.213 & -2467.6 & 0.9955 & 0.9974 & 0.0004 & 0.0003 \\
300 & 105.044 & -2316.2 & 0.9972 & 0.9984 & 0.0003 & 0.0002 \\
300 & 136.168 & -2156.2 & 0.9979 & 0.9991 & 0.0003 & 0.0002 \\
300 & 176.870 & -1949.4 & 0.9991 & 0.9995 & 0.0002 & 0.0001 \\
300 & 206.219 & -1802.1 & 0.9995 & 0.9997 & 0.0001 & 0.0001 \\
\hline
\end{tabular}

\begin{tabular}{|ccc|cc|cc|}
\hline \multicolumn{6}{|c|}{ Table S8. OPLS - AA } & \multicolumn{2}{c|}{ Uncertainty, $\boldsymbol{u}$} \\
\cline { 1 - 3 } $\boldsymbol{T}(\mathbf{K})$ & $\boldsymbol{P}(\mathbf{M P a})$ & $\boldsymbol{\mu} / \boldsymbol{k}_{b}(\mathbf{K})$ & $\boldsymbol{\theta}_{\text {Small }}$ & $\boldsymbol{\theta}_{\text {Large }}$ & $\boldsymbol{\theta}_{\text {Small }}$ & $\boldsymbol{\theta}_{\text {Large }}$ \\
\hline 300 & 0.100 & -4223.5 & 0.1102 & 0.1936 & 0.0019 & 0.0024 \\
300 & 0.200 & -4015.8 & 0.2139 & 0.3428 & 0.0025 & 0.0029 \\
300 & 0.501 & -3741.6 & 0.4407 & 0.6033 & 0.0031 & 0.0030 \\
300 & 1.004 & -3534.9 & 0.6343 & 0.7700 & 0.0030 & 0.0026 \\
300 & 2.023 & -3329.2 & 0.7846 & 0.8783 & 0.0025 & 0.0020 \\
300 & 5.127 & -3061.3 & 0.9043 & 0.9489 & 0.0018 & 0.0014 \\
300 & 10.526 & -2864.3 & 0.9466 & 0.9733 & 0.0014 & 0.0010 \\
300 & 21.669 & -2675.7 & 0.9723 & 0.9855 & 0.0010 & 0.0007 \\
300 & 52.265 & -2409.5 & 0.9882 & 0.9939 & 0.0007 & 0.0005 \\
300 & 76.664 & -2253.8 & 0.9928 & 0.9963 & 0.0005 & 0.0004 \\
300 & 99.085 & -2116.2 & 0.9958 & 0.9977 & 0.0004 & 0.0003 \\
300 & 127.483 & -1963.2 & 0.9972 & 0.9986 & 0.0003 & 0.0002 \\
300 & 165.699 & -1773.0 & 0.9987 & 0.9993 & 0.0002 & 0.0002 \\
300 & 193.667 & -1639.4 & 0.9991 & 0.9995 & 0.0002 & 0.0001 \\
\hline
\end{tabular}

